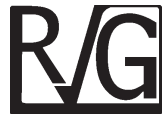

\title{
Gerencia del conocimiento: aproximación epistemológica en comunidades auto-eco-organizadas desde la perspectiva transcompleja
}

\author{
Guzmán, Jean Carlos*
}

\section{Resumen}

En la actualidad las sociedades se encuentran en un proceso de auto-transición como resultado de la conformación de la sociedad del conocimiento, que está incidiendo en todos los ámbitos, generando una serie de cambios en el entorno socio-eco-planetario. La Gerencia del Conocimiento permite la auto-generación de nuevas capacidades organizacionales así como la mejora de la efectividad, rendimiento y productividad en las comunidades auto-eco-organizadas. El objetivo de este trabajo es presentar una aproximación epistemológica al enfoque de gerencia del conocimiento en las comunidades auto-eco-organizadas venezolanas, desde la perspectiva transcompleja. La metodología utilizada consistió en una revisión bibliográfica especializada de las distintas nociones, concepciones, terminologías y conceptualizaciones del término gerencia del conocimiento desde la mirada de diferentes autores que están inmersos en diversas perspectivas epistemológicas. Como principal reflexión se tiene, la gerencia del conocimiento como enfoque transversal y transdisciplinario integra múltiples contextos, dimensiones y disciplinas, que requiere de una meta-visión y una meta-estrategia adecuada para alcanzar una gestión efectiva así como atractores y retractores para la conformación del tejido teórico reticular subyacente.

Palabras clave: Complejidad, transdisciplinariedad, transcomplejidad, gerencia del conocimiento, comunidades auto-eco-organizadas.

Recibido: 25-11-12 Aceptado: 15-01-14

Doctor en Ciencias, mención: Ciencias de la Computación. Profesor-Investigador adscrito al Departamento de Computación y Tecnología de la Información. Universidad Simón Bolívar. E-mail: jeanguzman@usb.ve 


\title{
Knowledge Management: An Epistemological Approach in Self-Organized Eco Communities from the Transcomplex Perspective
}

\begin{abstract}
Currently, societies are in a process of auto-transition as the result of configuring the knowledge society, which is affecting all spheres, generating a series of changes in the social-ecoplanetary environment. Knowledge management permits the self-generation of new organizational capacities as well as the improvement of effectiveness, performance and productivity in selforganized eco communities. The objective of this work is to present an epistemological approach to the focus on knowledge management in Venezuelan self-organized ecological communities, from the transcomplex perspective. The methodology consisted of a specialized bibliographic review of the different notions, concepts, terminologies and conceptualizations of the term knowledge management from the viewpoint of different authors who are immersed in diverse epistemological perspectives. A principal reflection is that knowledge management as a transversal and transdisciplinary approach integrates multiple contexts, dimensions and disciplines that require a meta-vision and a meta-strategy appropriate for achieving effective management as well as attractors and retractors for configuring the underlying reticular theoretical fabric.
\end{abstract}

Key words: Complexity, transdiciplinarity, transcomplexity, knowledge management, selforganized eco communities

\section{Introducción}

En la actualidad las sociedades se encuentran en un proceso de auto-transición como resultado del advenimiento de la era del conocimiento, que incide en todos los ámbitos y genera una serie de cambios en el entorno socio-eco-planetario (social, ecológico y planetario), los cuales son ocasionados por un conjunto de transformaciones que tienen como catalizador las Tecnologías de la Información y Comunicaciones (TIC), apoyadas por la educación académica como eje fundamental, cuyo impacto es transversal al respecto del entorno, en tanto que los grupos humanos como seres bio-psico-socio-eco-planetarios, están orientados a alcanzar la sociedad del conocimiento.
La Organización de las Naciones Unidas para la Educación, la Ciencia y la Cultura (Unesco, 2005: 18) al referirse a esta sociedad señala que "...es nutrida por la diversidad y sus capacidades..." cuyas dimensiones abarcan aspectos psico-económicos, socio-políticos, bio-éticos, eco-organizacionales y gerenciales desde una mirada dialo-holo-retroactiva (dialógica, hologramática y recursiva).

En este sentido, se considera al conocimiento como el nuevo recurso medular de la sociedad emergente, y se afirma que "...el factor productivo [luego de estar constituido] por el capital, la materia prima y la mano de obra, [ahora incorpora] a la información y al conocimiento" (Drucker, 1992: 13). Por tanto, se plantea que la información-conocimiento sea una generalización del referente histórico ca- 
pital-trabajo. De lo cual, se infiere que la producción y/o reproducción del conocimiento en esta etapa de socialización hacia la transmodernidad, transciende a la información, para posicionarse en una meta-visión que como recurso posibilita la emancipación del talento humano, hacia la sustentabilidad en las capacidades y la productividad de una entidad auto-eco-organizada, la cual es soportada por una meta-estrategia y un meta-proceso organizacional-gerencial. En este contexto, el adjetivo meta significa que el objeto (visión, estrategia o proceso) es de un nivel de abstracción superior, cuyos términos se definen a continuación:

Meta-visión es definida como una visión de nivel meta que proporciona los elementos, que especifican la visión de una comunidad organizacional, concebida como una imagen prospectiva anhelada para diseñar y construir el futuro desde el presente, significando una nueva realidad.

Meta-estrategia es percibida como una estrategia que agrupa o engloba otras estrategias, en la que se plantean diversos cursos de acción a un nivel macro, de forma que las opciones de decisión son estrategias per se, las cuales subyacen a un meta-proceso.

$Y$ meta-proceso, concebido como un proceso de nivel meta que es "responsable de la creación y la adaptación de un proceso" (Robertson, 1996: 2), por ende, está conformado por un conjunto de procesos y estos a su vez, por un conjunto de actores, actividades y tareas para ejecutar diferentes acciones en una organización orientada al conocimiento.

Bajo este preámbulos de ideas y dada la importancia del conocimiento para la sociedad, en los últimos años han surgido enfoques organizacionales centrados en la Gerencia del Conocimiento (GC) en los cuales se plantean estrategias y procesos de construcción asociados a la noción de modelos teóricos. La noción de modelo ha sido usada y desarrollada con la finalidad de explicar parte de la realidad desde diferentes miradas y matices, en cualquier modelo prevalece una teoría que lo sustenta y una acción que se concreta con una realidad verificable.

Graf (2004:11), define un modelo como "...una simplificación que imita los fenómenos del mundo real, de modo que se puedan entender las situaciones complejas y podamos hacer [representaciones de estas]". De allí que sean versiones mímicas que representan parte de la realidad, las cuales se constituyen en perspectivas epistemológicas, tales como: positivismo, fenomenología o complejidad desde una mirada filosófica donde se ubique el observador, como: premodernidad, modernidad, postmodernidad y transmodernidad.

Es necesario enfatizar que una perspectiva epistemológica, es un punto de vista "... teórico esencial en la que se puede incluir la investigación, tomando como base los objetivos y el marco teórico en que se sostienen sus premisas" (Araos, 2004:160). Según Suñé (2004: 166), para responder al posicionamiento, el investigador selecciona "...entre las perspectivas epistemológicas habitualmente identificadas como soporte a las Ciencias de la Organización". De allí que a partir de esas perspectivas, el investigador como sujeto, toma una posición o postura para encaminar su discurso y así 
analizar una situación objeto de estudio como la explicitada en el presente estudio, con la intención de develar, comprender, interpretar y resolver esa situación.

Bajo esta premisa, se considerada a la GC como un enfoque gerencial "...que permite a las organizaciones incrementar su valor al mejorar la forma en que crean, adoptan, validan, difunden, almacenan y usan el conocimiento" (Huircalaf, 2003: 6). Un enfoque transversal y transdisciplinario que integra múltiples contextos, dimensiones y disciplinas, que requiere de una meta-visión y una metaestrategia adecuada para alcanzar una gerencia efectiva así como atractores y retractores para la conformación del tejido teórico reticular subyacente.

Lo anteriormente expuesto, conlleva a una cosmovisión emergente que permite observar fenómenos que se suscitan en la realidad desde una perspectiva filosófica integradora. Una cosmovisión emergente de la naturaleza transcompleja, según Guzmán (2014: 118), es percibida como una "....meta-visión del mundo representada por la realidad bajo la figura de concepciones y nociones comunes para sujetos, época o cultura, en la que surgen atractores y retractores como elementos constituyentes", los cuales se definen a continuación.

Briggs y Pear (1989: 36), definen como atractor a la "... región del espacio de fases que ejerce una atracción magnética sobre un sistema, y parece arrastrar el sistema hacia él". Son zonas dinámicas que pueden ser definidas como áreas de atracción, donde se desenvuelve un conjunto de elementos o variables atípicas o emergentes al contexto en el cual se desempeñan. Por ende, son de gran relevancia para el investigador por su alto valor agregado en la construcción y reconstrucción del conocimiento.

En contraste, un retractores un elemento o variable que se aleja de la zona de atracción (campo magnético), transitando hacia una zona borrosa y difusa que limita su comportamiento en aparente orden. Según Kosko (1995: 5), esto se corresponde a una "...característica determinada, sobre el universo de objetos que la conforman", cuyo valor representa el estado con el que dicho objeto se presenta en una realidad en particular. De allí que, la borrosidad aluda a la imprecisión o alejamiento de un elemento o variable de los límites de esa realidad, la cual se constituye en una zona que ostenta fronteras permeables, desde el punto de vista transcomplejo.

La transcomplejidad surge de la crisis científica actual, producto de la existencia de varias perspectivas filosóficas en el ámbito científico. Los planteamientos de esta perspectiva epistemológica retan al pensamiento clásico de la racionalidad como transición del pensamiento de la simplicidad hacia un pensamiento complejo. Surge por los avances de la electrónica, cibernética, informática, matemáticas y revolución científico-técnica, así como por los problemas epistemológicos y prácticos no resueltos por las perspectivas clásicas de la filosofía. Lo cual implica según González (1997: 61):

"Aceptar la naturaleza múltiple y diversa de lo estudiado, la integración y desintegración de elementos diferentes y contradictorios en distintos tipos de unidad, la aceptación del cambio y la mutabilidad de los objetos, de lo imprevisto como forma de expresión alternativa de 
un sistema ante hechos similares ocurridos en el tiempo, así como comprender formas irregulares de orden, rompiendo con el concepto de orden equivalente a secuencia regular".

Desde esta óptica, la transcomplejidad como episteme, desafía la reelaboración de nuestras categorías mentales desechando como reglas el historicismo, la fragmentación y la simplificación del conocimiento. Se centra en el homocomplexus como ser bio-psico-eco-planetario dirigido hacia la construcción y reconstrucción del conocimiento, como elemento significativo para avanzar hacia una sociedad transcompleja. Es una perspectiva epistemológica emergente de la racionalidad con parámetros fundados en incertidumbre e indeterminación, antagonismo y complementariedad, unidad y diversidad, potencialidad y devenir como abordaje requerido para el estudio de la GC en las comunidades auto-eco-organizadas venezolanas, la cual involucra en su abordaje varias disciplinas de diferentes niveles de abstracción y dominios que permiten a la gestión alcanzar sus objetivos y metas desde el punto de vista organizacional-gerencial transcomplejo.

La racionalidad en este contexto, se constituye en la racionalidad de lo complejo, diverso, múltiple, plurar y transversal. Según Silva (2014: 76), se caracteriza por ser "...esencialmente transitiva, transeúnte, transida de la racionalidad compleja: como razón transversal"; que exige la transformación de la manera de pensar, la constitución y sus funciones.

El objetivo de este trabajo es realizar una aproximación epistemológica al enfoque de gerencia del conocimiento en las comunidades auto-eco-organizadas venezolanas, desde la perspectiva transcompleja. El mismo es de gran relevancia para el estudio de la GC en las Organizaciones Venezolanas y para la conformación de su tejido teórico-reticular desde esta perspectiva epistemológica.

La perspectiva epistemológica adoptada, en atención a la naturaleza del objeto de estudio del presente trabajo, se corresponde a la perspectiva transcompleja, en vista que se constituye en un episteme emergente que integra la complejidad y la transdisciplinariedad para ubicarse en contexto transparadigmático dirigido hacia la ecología del conocimiento, la multiversidad de la realidad, las estructuras rizomáticas y la integración de atractores y retractores al tejido teórico reticular en su conjunto.

La metodología utilizado, consistió en una revisión bibliográfica especializada de las diferentes nociones, concepciones, terminologías y conceptualizaciones desde la mirada de diferentes autores que están inmersos en diversas perspectivas epistemológicas, procedente de la literatura disponible y relacionada con el objeto de este estudio.

\section{La gerencia del conocimiento}

En los últimos años han surgido enfoques organizacionales en el ámbito gerencial, tales como la Gerencia del Conocimiento (GC), el cual permite a una organización la auto-generación de sus capacidades así como la mejora de su efectividad, rendimiento y productividad desde una perspectiva organizacional-gerencial. Como consecuencia de ello, se han percibido las bondades que brinda y trae 
consigo el referido enfoque gerencial, debido al ahorro sustancial de recursos financieros como resultado de la mejora de procesos, productos y servicios en las comunidades organizacionales, los cuales se traducen en altos índices de rendimiento, debido a la incorporación de programas de GC, como estrategia que garantiza la sustentabilidad en una autoeco-organización.

Estos trabajos manifiestan que el mundo actual, está inmerso en un proceso de socialización motorizado por las TIC, donde el conocimiento transciende a la información. Un proceso de cambio optimista orientado hacia la denominada Sociedad del Conocimiento, en la cual el conocimiento es considerado un recurso medular que al ser gestionado adecuadamente, permite la construcción de una organización focalizada en la innovación de productos y servicios, con el objeto de generar capacidades organizacionales y así mantenerse sustentable en el tiempo.

Para Davenport y Prusak (2001: 7), el conocimiento "... puede y debe ser evaluado mediante decisiones o medidas a las que conduce. Podemos usarlo para adoptar decisiones más sensatas sobre la estrategia". Bajo esta premisa, se plantea que la GC es un enfoque gerencial que está constituido por herramientas, métodos, técnicas y procesos que al ser gerenciados adecuadamente, permiten agregar valor y alcanzar objetivos y metas desde una perspectiva organizacional-gerencial. Un enfoque que aporta estrategias y procesos transversales a las organizaciones debido a su incidencia en otros procesos adelantados en éstas.
Según Guzmán (2010:8), la idea es "...centralizar y difundir el conocimiento tácito, poniéndolo a la disposición de quienes lo necesiten, en un ambiente colaborativo necesariamente compartido donde interactúen personas pertenecientes a una entidad en particular", el cual es concebido como aquel conocimiento orientado por la experiencia de un individuo que se manifiesta en competencias y destrezas, adquiridas e internalizadas a través del tiempo, que se exteriorizan en diferentes miradas y matices como resultado de sus autoconstrucciones al retrotraerse en un entorno socio-eco-planetario.

Cabe señalar que el conocimiento perteneciente al homocomplexus forma parte de su talento, el cual es considerado una agregación compleja de significados que ha logrado internalizar al retroactuary retrotraerse con las cosas u objetos del mundo real. Sin embargo, tales autoconstrucciones son transferidas entre los actores sociales que conforman una comunidad auto-eco-organizada mediante la comunicación, praxis ésta que permite entre otras cosas, compartir e intercambiar conocimientos de manera colaborativa siempre y cuando la comunicación sea dialógica y consensual.

Estas aproximaciones constituyen el enfoque gerencial emergente de las auto-eco-organizaciones orientadas al conocimiento, el cual está centrado en la sinergia, la colaboración y la cooperación efectiva de los actores sociales con el propósito de satisfacer objetivos y metas de manera colectiva en un entorno socio-eco-planetario. 


\section{Comunidades auto-eco-organizadas}

Según Velilla (2002: 17), la transcomplejidad puede ser visualizada en “...relación con la evolución o el cambio de los sistemas vivos... como la capacidad del sistema para auto-organizarse de manera emergente, en su relación negantrópica con el entorno". Es percibida como la emergencia del orden en los entornos que tiende al desorden y está asociado a la dialéctica sistema-organización.

Para Soto (1999), el concepto de sistema y de organización está mediado por el concepto de interrelación, en tanto que un sistema es una unidad global compleja, organizada y organizadora, de interrelaciones entre lo múltiple y lo diverso que lo constituye, posee cualidades nuevas, propiedades emergentes 0 atractores que son irreductibles a las propiedades de sus componentes, consideradas de manera aislada o yuxtapuesta.

La organización concebida de esta manera, se plantea como "... la relación de las relaciones, forma lo que transforma, transforma lo que forma..., se organiza al organizar y organiza al organizarse" (Morín, 1977: 136). Es una disposición activa que, siendo producto de las partes, emerge de sus interrelaciones, pero actúa sobre ellas, de modo que las transforma, produce, reúne y mantiene. En efecto, establece relaciones complementarias entre partes diferentes-diversas y entre ellas y el todo.

Al hablar de auto-eco-organizaciones, Morín (1980: 46), señala que la “...eco-organización necesita de la autoorganización (y ésta a su vez necesita de la eco-organización); autos aporta al oikos conocimiento, computación, comunicación, estrategia, reproducción, un frenético querer vivir, y todo esto, vía las interacciones, irriga la espontaneidad ecoorganizadora".

El término griego oikos designa la casa, el hábitat, y da origen a ecología. Se trata de una realidad sistémica-organizada, que supera los principios de organización estrictamente físicos. Mientras que la eco-organización es "...una organización a la vez física y viviente cuya originalidad está en su carácter viviente que, ... retroactúa sobre su carácter físico" (Morín, 1980: 46). Entendiéndose por retroacción, al bucle recursivo como noción de regulación y mantenimiento, lo cual admite la iteración de un sistema sobre sí mismo; produce los elementos y efectos necesarios a su propia generación o existencia.

El término griego autos forma parte como prefijo "auto" para significar por sí o de sí mismo. Para Morín (1980: 108), la palabra pretende ser nada menos "la palabra-esfinge que nos plantea el gran enigma de la vida". Las ideas de auto-eco-organización, según Velilla (2002: 17), nacen al concebir a "... todo sistema en su condición de propietario de algunos mecanismos selectivos a través de los cuales se desarrolla una activa capacidad de respuesta frente a su ambiente". Estas ideas fueron retomadas y reformuladas al enfatizar la capacidad de autonomía y de auto-organización de algunos sistemas, incorporando a la teoría, las nociones de auto-referencia, auto-observación, reflexión y autopoiesis son asumidas en este trabajo bajo la noción de comunidades auto-eco-organizadas, las cuales son de- 
finidas como organizaciones vivas, dependen del entorno para obtener energía e información. Se constituye en la base de una organización que trabaja para auto-mantenerse, consume energía en sus labores, por lo que debe obtener energía de su entorno.

Las comunidades organizadas como auto-eco-organizaciones subyacen a procesos continuos de cambios en su estructura a lo largo de su vida, se correlacionan a la adaptación y a las transformaciones suscitadas en el entorno, en un contexto determinado. Gil y Toba (2008: 70 ), plantean que la transcomplejidad inserta en estos contextos sus “... principios para apoyar de manera clara y contundente la noción de que se está dando el salto de la auto-organización a la denominada auto-eco-organización"; en la que se plantea a las comunidades organizacionales, como seres vivientes, en el que inter-retroactúa el entorno natural y social.

\section{La perspectiva transcompleja ${ }^{1}$}

En los últimos años se ha ido autoconformando una eco-cognición que integra las perspectivas de la complejidad y transdisciplinariedad, la cual trasciende la episteme cartesiana de la ciencia y, de hecho, la auto-redefine en una orientación transcompleja. Al respecto, Vergara (2010: 11), señala que la episteme de la transcomplejidad es usada“...para inte- grar los planteamientos del paradigma de la complejidad y de su método de estudio, la transdisciplinariedad".

De lo que antecede, Oliveros (2013: 103), señala que la concepción eco-cognitiva puede ser interpretada como un episteme "...cultural en cuyo discurso las palabras clave son: sobrevivencia, conciencia, ecología y acción conjunta".

Por otra parte, Morín (2003: 2), señala que complejo es aquello que "...no puede resumirse en una palabra maestra, aquello que no puede retrotraerse a una ley, aquello que no puede reducirse a una idea simple". Dicho de otro modo, lo complejo no puede resumirse en el término complejidad, retrotraerse a una ley de complejidad, reducirse a la idea de complejidad. Sin embargo la transdisciplinariedad, según Nicolescu (1999: 35), le concierne como "...el prefijo trans lo indica, lo que está a la vez entre las disciplinas, a través de las diferentes disciplinas y más allá de toda disciplina".

De allí que la transcomplejidad se encuentra contenida en diversos metadominios del conocimiento donde confluyen psicología, espiritualidad, antropología, política, lingüística, ecología, economía, historia y filosofía, entre otras ciencias. Para Unesco (1997: 2), la transcomplejidad es una: Nueva visión y de una experiencia vivida. Es una vía de autotransformación orientada hacia el conocimiento y hacia la creación de un nuevo arte de vivir...la investigación bajo [esta 
perspectiva] debe adecuarse a problemas transversales, transcendentales y multidimensionales como las meta-meta-teorías.

Desde esta concepción, se plantea que el objeto de investigación debe necesariamente concebirse como apertura a espacios de múltiples miradas y matices dando paso una perspectiva multiversa. Según Maturana (1988), el observador bajo esta perspectiva, se "...reconoce como un participante constitutivo de lo que observa... cada mundo construido por el observador es igualmente válido y único respecto de otros." Para Schavino y Villegas (2010: 4), la investigación transcompleja es una nueva cosmovisión que propugna: "La adopción de una posición abierta, flexible, inacabada, integral, sistémica y multivariada, donde lo cuantitativo, lo cualitativo y lo dialéctico se complementan en una relación sinérgica que configura una matriz epistémica multidimensional. Es entonces, un proceso bioafectivo cognitivo, pero también sociocultural-institucional-político de producción de conocimientos, como un producto complejo que se genera de la interacción del hombre con la realidad de la cual forma parte".

De esta manera la transcomplejidad en el ámbito científico tiene por finalidad, la comprensión del mundo presente desde el imperativo de la complementariedad del conocimiento. Su interés es la "... dinámica de la acción y se apoya en la existencia y percepción de distintos niveles de la realidad, en la aparición de nuevas lógicas y en la emergencia de la complejidad" (Schavino y Villegas, 2010: 4). Bajo este orden ideas, la transcomplejidad se muestra como una perspectiva in- tegradora que busca indagar y descubrir múltiples manifestaciones en los fenómenos que se manifiestan en la realidad.

Tal y como lo expresa Lanz (2001: 30), se dirige a: "Trascender en el pensamiento, sin barreras disciplinarias, sin esquemas universales, sin escisiones entre lo natural y lo humano, sin la superioridad de lo cuantitativo apoyado en la medición, sin exclusión de la paradoja, sin execrar a la poesía o a cualquier otra dimensión del arte, sin sustitución del diálogo por las pruebas teóricas o empíricas, en fin, sin sacrificar la totalidad del mundo incluida su armonía estética".

Para Moreno (2002:127), la epistemología transcompleja "...traduce la crisis paradigmática y la complejización de la epistemología, en un pensamiento epistemológico articulador, plural y fluido", que se organiza-desorganiza y reorganiza. Es un nuevo modo de "... producir conocimientos transdisciplinarios..." (Villegas, 2006) que va más allá de la complejidad para ubicarse en contexto transparadigmático, dirigido hacia la ecología del conocimiento, la multiversidad de la realidad, las estructuras rizomáticas y la integración de atractores al tejido en su conjunto. En tanto que la Rizomática, según Deleuze y Guattari (1994:19), se plantea como: "Una eco-cognición dirigida a las relaciones y conexiones transversales, que vinculan el conocimiento complejo con el enfoque transdisciplinario, sin anclarse ni en uno ni en otro. Un rizoma es un intermediario que siempre está en el medio, entre las cosas, interser e inter-mezzo".

Por consiguiente, la transcomplejidad es un transparadigma en el que, según Fernández (2006: 40), cohabita: "Lo 
lógico y lo paradójico, lo racional y lo irracional, el azar y lo necesario, la coherencia y la paradoja, lo lineal y lo no-lineal, el orden y el desorden, la certidumbre y la incertidumbre, en relaciones abiertas, complejas y de complementariedad para asumir, sin tributo alguno a viejos esquemas unidimensionales, reduccionistas y mutilantes, la tarea desgarrada y, a la vez fecunda que implica el acto o producción del conocimiento, pero ahora en un campo siempre abierto, despejado de teologías y fundamentalismos y, por ello, dotado de inmensa posibilidades".

De allí que la perspectiva epistemológica sea un punto de vista del pensamiento, sin restricciones disciplinarias, esquemas universales, escisiones entre lo natural y lo humano, superioridad de lo cuantitativo apoyado en la medición, exclusión de la paradoja, sustitución del diálogo por las pruebas teóricas o empíricas, en síntesis, sin sacrificar la totalidad del mundo incluida su armonía estética.

Para Lanz (2000:10), la ciencia en el marco de esta perspectiva: "Es una ciencia de la complejidad... que juega a lo múltiple, al pluralismo de la razón, a la infinita variedad de la vida. Diversidad ecológica, diversidad cultural, diversidad intersubjetiva, diversidad estética. Una ciencia del caos y la indeterminación que rompe con toda linealidad y con los modelos causalistas del pasado. Una ciencia de las verdades que puede jugar con distintas racionalidades...que se define heterogénea desde el punto de partida..., una ciencia transdisciplinaria que rompe definitivamente con los encierros de la lógica de las disciplinas y abre el conocimiento a la inmensidad de las interpene- traciones, dando lugar a nuevos territorios poblados de nuevas preguntas".

Desde esta concepción, las restricciones quedan abolidas $\mathrm{y}$, el aspecto de rizoma inherente a la naturaleza es retomado para la construcción y reconstrucción del conocimiento. Por tanto, se plantea que la ciencia es subsumida e integrada en una eco-cognición transcompleja, junto a sus métodos y procedimientos. Es así que para resolver una situación problemática en el marco de esta perspectiva, se requiere una descripción contextualizada e integradora, en relación a las etapas precedentes y a sus potencialidades de continuidad.

Bajo esta premisa, la transcomplejidad como aspiración socio-eco-planetaria (social, ecológica y planetaria), según Gil y Toba (2009), abre nuevos “...espacios para la reflexión y la comprensión del hecho organizacional, así como también para el cambio paradigmático en el pensamiento humano". Permitiendo la sinergia, la sumatoria exponencial de los esfuerzos, así como organizaciones verdaderamente interrelacionadas en un tejido, en la búsqueda de una auto-eco-evolución.

En tanto que se interpreta, según Vergara (2010: 36): "Una conexión con lo histórico y desde una opción de futuro, con énfasis en la posibilidad innovativa, inventiva, auto-transformativa de los sujetos, donde se incluya la posibilidad de aparición de ramificaciones, asociadas al azar y a cualidades emergentes, rumbos nuevos, impulsados por elementos extraños, que no pueden ser establecidos desde el inicio del proceso... Es necesario reconstruir una visión flexible de lo social y 
de las formas de intervención en el cambio que incorpora necesariamente la incertidumbre y la subjetividad como fuerzas que intervienen en el curso de los acontecimientos".

De allí que se haga impostergable considerar bajo esta perspectiva: lo humano, tecnológico, económico, político, ético y estético, la afectividad y creatividad, lo normativo y costumbres, la intuición y espiritualidad, la experiencia y experticia del investigador y participación de los actores del proceso, donde se integra el conocimiento científico, filosófico y cotidiano, con la utilización de múltiples-métodos en el desarrollo de una investigación. Schavino y Villegas (2010: 4-7), señalan siete (7) principios de la investigación transcompleja, entre las cuales destacan:

- Principio de complementariedad: Asume la complementariedad investigativa, como una nueva tendencia, fundamentada en la aplicación de metodologías transdisciplinarias, que permitan tanto la comprensión de las diferentes vertientes de un problema, así como de posibles soluciones a los mismos, y las consecuencias que a partir de sus aplicaciones se llegaran a desencadenar.

- Principio epistemológico: Hace referencia a una nueva concepción de racionalidad científica, que conduce a la superación de las antinomias, las paradojas y las aporías, y que ponga de relieve el carácter complementario y transdisciplinario que enmarcan y constituyen el contexto ontológico en el que se desempeña la labor de investigar.
- Principio de sinergia relacional: Conduce a la idea de unidad y supone la renuncia a la individualidad en pro del fortalecimiento del colectivo.

- Principio de consenso: Encuentro de visiones, de paradigmas, de posturas y de consenso dialéctico.

- Principio de integralidad: La integralidad trasciende al holismo y denota la necesidad de asumir que la realidad es múltiple, diversa, relacional, en construcción y por ello, también construible, abriendo un camino a lo interaccional, a lo reticular.

- Principio de reflexividad: La reflexividad es un proceso complejo de deliberación del pensamiento sobre la interpretación de una experiencia para poder aprender de ella, abre las fronteras entre las ciencias humanas y las ciencias naturales, permitiendo generar convergencias.

- Principio de universalidad: Asume el déficit de complementariedad con lo local y lo singular; reinserta el tiempo irreversible en los fenómenos de la naturaleza y del universo; hace juego con un principio discursivo complejo donde habitan lo complementario y lo contradictorio, integra la borrosidad en la inteligibilidad de los fenómenos $y$, por tanto las apreciaciones de grado y aproximación.

Los principios que anteceden, se constituyen en un marco conceptual de referencia que guía al investigador en el abordaje del objeto de estudio bajo esta perspectiva epistemológica. Sus principios y características son fundamentales para abordar la gerencia del conocimien- 
to en las comunidades organizacionales como sistemas auto-eco-organizados.

\section{Gerencia del conocimiento: comunidades auto-eco-organizadas desde la transcomplejidad}

La gerencia en las auto-eco-organizaciones ha sufrido una serie de transformaciones en las últimas décadas. Es imprescindible enfatizar que tales transformaciones han incidido en el solapamiento entre las diferentes perspectivas epistemológicas que han emergido en el tiempo y que han ido auto-construyendo las Ciencias Gerenciales. Solapamiento ese que se constituye en un andamiaje entre y a través de las citadas perspectivas, de modo que un enfoque organizacional-gerencial soporta o sustenta al siguiente, pero no lo extingue por completo sino que lo auto-redefine o lo auto-configura en el tiempo; adaptándolo a las exigencias de los actores socio-eco-planetarios (sociales, ecológicos y planetarios), lo cual propende a una perspectiva epistemológica re-integradora como la transcomplejidad.

En este sentido, las tendencias organizacionales desde la perspectiva transcompleja están supeditadas a cambios, turbulencias, incertidumbres e indeterminaciones experimentadas, en escenarios de situaciones y problemas complejos que se confluyen mutuamente, obligando a que las decisiones cotidianas sean por excelencia auto-transformadoras.

Mientras tanto, el ámbito organizacional ha trascendido de lo local a lo nacional y de éste a lo global, auto-confor- mando una nueva concepción bajo la dialéctica organización-red-mundo y su vinculación auto-eco-planetaria (autónoma, ecológica y planetaria). Una cosmovisión emergente en el ámbito organizacionalgerencial desde la transcomplejidad admite teóricamente, el desarrollo de alianzas entre y a través de las comunidades auto-eco-organizadas a nivel local, regional y global; y una visión orientada hacia los servicios, en la cual el consumidor ha sido transformado en la pieza central de la economía, donde la lógica de la demanda sustituye la lógica de la oferta. Aunado al hecho que las estructuras de estas comunidades organizacionales han trascendido de una arquitectura jerárquica (positivista) a una arquitectura matricial (fenomenológica) y de una arquitectura matricial a una arquitectura en redes (compleja) dada la supresión de sus fronteras, que se corresponden a las transformaciones que propicia la denominada transdisciplinariedad.

Por otro lado, existe un acelerado incremento en el comercio internacional, una mayor competitividad, necesidades de alianzas, dependencia tecnológica, terciarización de la economía y surgimiento de redes altamente calificadas. Lo cual se acompaña de una permanente y acelerada construcción y reconstrucción del conocimiento científico y tecnológico que se manifiesta en innovaciones y desarrollos de productos y servicios, cuya aplicación a la producción y gestión organizacional le imprimen un escenario transcomplejo, que imperiosamente debe ser visualizado desde una perspectiva multiversa.

Los enfoques organizacionales no escapan a estos escenarios que se co- 
rresponde a la variabilidad suscitada en el entorno socio-eco-planetario dado que están sujetos a un proceso de auto-transformación mediante el cual se adecuan a escenarios que plantea la eco-sociedad, dado la existencia de: primero, diversidad de intereses, compromisos, preocupaciones y necesidades que actúan sobre la misma auto-eco-organización, incidiendo en diferentes sentidos desde una perspectiva dialógica; segundo, incorporación de arquitecturas auto-eco-organizacionales en redes; tercero, tendencia a la auto-constitución de nuevas eco-culturas en distintas ámbitos, áreas y niveles ecoestructurales, con sus propias espiritualidades, creencias y costumbres de la realidad compleja; cuarto, tendencia hacia la auto-conformación de comunidades auto-eco-organizadas con presencia localregional y global; quinto, amenaza de competidores que se mueven en los ecomercados abiertos y foráneos utilizando eco-estrategias ofensivas hacia la autoeco-organización; sexto, reducción de plazos en la renovación de la eco-tecnología y de los ciclos de vida de los productos y servicios; séptimo, creciente información disponible en internet. Y, octavo, auto-eco-organizaciones dirigidas hacia la gerencia del talento humano.

Por tanto, se hace impostergable la construcción de un enfoque de GC fundamentado en una perspectiva basada en infinitas interacciones y múltiples reconstrucciones auto-generadoras, que lo auto-redefina o auto-conforme.

Bajo esta premisa, se plantea la existencia de elementos que habilitan la GC tales como cultura, arquitectura organizacional, tecnología y valoración; los cuales son considerados elementos im- prescindibles en la implementación de este enfoque en una comunidad auto-eco-organizada.

Sin embargo, desde el punto de vista de la transcomplejidad se plantea un conjunto de atractores que actualmente no han sido incorporados como elementos habilitadores para alcanzar una verdadera GC, entre los cuales se tiene: antropología, política, lingüística, ecología, economía, historia, filosofía, ética, psicología, espiritualidad, entre otros. Estos atractores conforman una nueva cosmovisión organizacional-gerencial orientada hacia el conocimiento como un recurso medular para las comunidades auto-eco-organizadas.

En necesario enfatizar que la autoredefinición o auto-conformación de un nuevo enfoque de GC desde esta perspectiva, requiere de un método emergente que contemple estrategias, planes y procesos dirigidos hacia su auto-construcción. Por consiguiente, este enfoque permitiría redefinirse en una eco-cultura encaminada hacia el cambio, la participación y el protagonismo de los actores socio-eco-planetarios que intervienen en los diferentes procesos adelantados en las comunidades organizacionales, desde donde nace la necesidad de autoconstruir y reconstruir conocimientos sobre la base de experiencias y prácticas aprendidas en el desarrollo de las actividades y tareas, hace indispensable el intercambio, la colaboración y la cooperación para la consecución de objetivos y metas emanadas por la gestión.

En este sentido, se plantean cinco (5) elementos estratégicos que deben ser considerados en la auto-redefinición del enfoque de GC en las comunidades au- 
to-eco-organizadas en Venezuela desde la transcomplejidad. Primero, es un proceso bio-psico-eco-social y espiritual en el que los sujetos sociales interactúan y comparten conocimientos en una realidad determinada de la cual forman parte, superando las disyunciones sujeto-objeto, y abriendo camino a lo interaccional y a lo reticular, como fuentes constitutivas. Segundo, con los conocimientos (aprendizajes, saberes y haceres) compartidos se obtienen mayores y mejores resultados, materializando objetivos implícitos o explícitos como aspiración socio-eco-planetaria, se presenta en un agente catalizador de los procesos adelantados en las comunidades auto-eco-organizadas. Tercero, está soportado por personas quienes son agentes críticos de éxito y fundamentales para garantizar la calidad lo que se constituye en un aspecto complejo, multidimensional, integrador, comprensivo, ecológico y rodeado de incertidumbre dentro de las auto-eco-organizaciones. Cuarto, el conocimiento se construye y reconstruye en el individuo y forma parte de sus competencias como agregación constituyente del talento humano generalizado como propiedad intelectual colectiva en las comunidades auto-eco-organizadas, lo cual se instaura en el potencial hacia el desarrollo e innovación de productos-servicios para garantizar la satisfacción de necesidades presentes y futuras. Y quinto, el conocimiento es libre, otorgándose libertad para ser utilizado, redistribuido, mejorado y compartido sin restricciones, con miras a ser utilizado y mejorado, lo que permite incrementar a nivel individual las competencias de los sujetos sociales y a nivel colectivo las potencialidades de un siste- ma transcomplejo como son las comunidades auto-eco-organizadas venezolanas.

\section{Reflexiones finales}

En la actualidad existen tendencias científicas encaminadas a resolver los problemas epistemológicos existentes en las Ciencias Sociales, particularmente en las Ciencias Gerenciales desde donde nace la necesidad del presente estudio, abordado a través de la perspectiva de la transcomplejidad.

La transcomplejidad es una perspectiva epistemológica emergente que plantea un nuevo modo de percibir y hacer ciencia que va más allá de la perspectiva de la complejidad, la cual tiene la posibilidad de permitir la auto-construcción y reconstrucción de nuevos escenarios científico-académicos, emocionales-intelectuales y ético-morales, distintivos que transcienden las perspectivas que le anteceden.

Las ideas de la transcomplejidad como cosmovisión emergente está generando cambios profundos en la manera de comprender la relación parte-todo; determinismo-indeterminismo, caos-ordendesorden implícitas en los sistemas del caos y el anti-caos; una fuerte tendencia hacia la superación de la perspectiva epistemológica del positivismo en la filosofía de las ciencias con el amalgamiento de la fenomenología y la complementariedad de sus métodos desde la dialéctica cuantitativo-cualitativo, así como en el modo de concebir la inter-retroacción del homocomplexus en un entorno socio-eco-planetario. Se define como un sersensible, neurótico y delirante, pero particu- 
larmente racional, constituido por un tejido propio de los humanos, cuyos conocimientos internalizados (aprendizajes, saberes y haceres) en una realidad determinada donde interactúa, son exclusivamente compartidos; constituye el punto central sobre el cual gira toda comunidad auto-eco-organizada desde una mirada organizacional-gerencial emergente.

La Gerencia del Conocimiento (GC) es un enfoque que está constituido por herramientas, métodos, técnicas y procesos que permiten generar valor y alcanzar objetivos y metas desde una perspectiva organizacional-gerencial. Un enfoque transversal y transdisciplinario que integra múltiples contextos, dimensiones y disciplinas, que requiere de una metavisión y una meta-estrategia adecuada para alcanzar una gerencia efectiva así como atractores y retractores para la conformación del tejido teórico reticular desde la transcomplejidad.

La cual se manifiesta, en diversidad de productos o servicios a gerenciar, en cadenas de creación de valor. Y por ende, se habla de complejidad cuando los nudos de dirección superior de estos sistemas se enfrentan con tareas de organización y coordinación, planificación y control o revisión... fenómeno habitual tras fusiones decididas por aparentes razones de sinergia entre empresas con líneas de creación de valor muy distintas".

En este trabajo se realizó una aproximación epistemológica al enfoque de gerencia del conocimiento en las comunidades auto-eco-organizadas desde la perspectiva transcompleja. Es por ello que se hace impostergable la auto-redefinición o auto-conformación del citado enfoque desde una perspectiva epistemoló- gica basada en infinitas interacciones y múltiples reconstrucciones auto-generadoras. Esto se logra asumiendo los principios y características fundamentales de la transcomplejidad e incorporando un método emergente que integre estrategias, planes y procesos dirigidos hacia su auto-construcción y reconstrucción.

Lo cual permitiría la conformación de una eco-cultura encaminada hacia el cambio, la participación y el protagonismo de los actores bio-psico-socio-ecoplanetarios que intervienen en los diferentes procesos adelantados en las comunidades auto-eco-organizadas, desde donde nace la necesidad de construir y reconstruir conocimientos sobre la base de experiencias y prácticas aprendidas en el desarrollo de las actividades, lo cual hace indispensable el intercambio, la colaboración y la cooperación para la consecución de objetivos y metas emanadas por la gestión.

En trabajos futuros se definirá un conjunto de principios ontológicos para el posicionamiento del sujeto ante la naturaleza de una realidad transcompleja como la exteriorizada en el presente estudio.

\section{Referencias bibliográficas}

Araos, Carlos (2004). Análisis documental de los aspectos teórico-metodológicos de la investigación chilena sobre medios de comunicación. Revista Científicas Complutenses. Documentación de las Ciencias de la Información, vol. 27, 157-218.

Briggs, John y Pear, David (1989). Turbulent mirror: an illustrated guide to chaos theory and the science of wholeness. New York, EUA. Harper Perennial. 
Davenport, Thomas y Prusak, Laurence (2001). Conocimiento en Acción: Cómo las organizaciones manejan lo que saben. Buenos Aires, Argentina. Pearson Education S.A.

Deleuze, Gilles y Guattari, Félix (1994). Mil mesetas. Valencia, España. Pre-textos.

Drucker, Peter (1992). La sociedad poscapitalista. Barcelona, España. Apóstrofe.

Fernández, Ángel (2006). Epistemología transcompleja. [Online]. Disponible en: http://debatecultural.org/Observatorio/AngelAmericoFernandez2.htm. [Consultado en: Julio de 2011].

González, Fernando (1997). Epistemología Cualitativa y Subjetividad. La Habana, Cuba: Pueblo y Educación.

Guzmán, Jean Carlos (2010). Modelo de gerencia de proyectos para la Gerencia de Automatización, Informática y Telecomunicaciones de Pdvsa, desde la Gestión Social del Conocimiento. Trabajo de Grado. UNEFA, Caracas-Venezuela.

Guzmán, Jean Carlos (2014). Cosmovisión emergente de la naturaleza de la realidad desde la perspectiva de la transcomplejidad. Proc. II Jornada de Investigación Tecnología y Transcomplejidad: Avances y Prospectivas, Maracay, Estado Aragua, Venezuela, 05 y 06 de junio.

Gil, Ricardo y Toba, Consuelo (2009). Hacia una Teoría de los modelos organizativos transcomplejos como aspiración socio-eco-planetaria. Revista Visión Gerencial, Año 8, № 1, Enero-Junio 2009, Pg: 63-73.

Graf, Esteban (2004). El Abordaje de la Realidad a través del Enfoque de Sistemas. [Online]. Disponibilidad: www. fagro.edu.uy/ ambiental/ecologia/ docs $/ 11 \% 20$ Graf $\% 202004 \% 20$ el $\% 20$ enfoque $\% 20$ de $\% 20$ sistemas.pdf. [Consultado: junio de 2014].
Huircalaf, Juan (2003). La revolución del conocimiento en la sociedad y la empresa. [Online]. Revista Gerencia. Disponible en: http://www.emb.cl/gerencia/ articulo.mv? sec $=10 \&$ num $=9 \& \mathrm{mag}=1$ $\&$ wmag $=25$. [Consulta 27 de marzo de 2014].

Lanz, Rigoberto (2000). De la ciencia, Nietzsche y otros extravíos. Revista Latinoamericana de Estudios Avanzados, Número 11, Caracas, 2000, pp.10-11.

Lanz, Rigoberto (2001). Diez tesis sobre la cultura organizacional transcompleja. Procesos Organizaciones transcomplejas. Caracas: Editorial Imposmo/Conocit.

Maturana, Humberto (1988). Ontology of Observing: The biological foundations of self-consciousness and the physical domain of existence. Conference Workbook: Texts in Cybernetics, American Society for Cybernetics Conference, Felton, CA. 18-23 October.

Moreno, Juan Carlos (2002). Complejización de la epistemología y epistemología compleja. [Online]. Disponible en: http://www.pensamientocomplejo. com.ar/docs/files/Vilella_Manual_pedagogia_pc.pdf. [Consultado en: julio de 2011].

Morín, Edgar (1977). El método I: La naturaleza de la naturaleza. Seuil, Paris. Trad. Ana Sanchez, Catedra, Madrid.

Morín, Edgar (1980). El método II: La vida de la vida. Seuil, Paris. Trad. Ana Sanchez, Catedra, Madrid.

Morín, Edgar (2003). Introducción al pensamiento complejo. España: Gedisa.

Nicolescu, Basarab (1999). Transdisciplinaritatea: The Manifesto of Transdisciplinarity, Polirom Publishing, lasi, 1999 (translated in Romanian by Horia Mihail Vasilescu), (second Romanian edition: Junimea Publishing, lasi, 2007). 
Oliveros, Rosa (2013). Del latido al byte sección para compartir saberes tecnológicos y educativos enmarcados en la educación a distancia. UNAVISION, 109-110.

Robertson, Ian (1996). An implementable meta-process. In Second World Conference on Integrated Design and Process Technology.

Rodriguez, José (2005), Epistemología de la complejidad. CEPADE. Universidad Politécnica de Madrid. [Online]. Disponible en: www.ub.edu/iafi/Recerca/ Seminaris/complejidad.doc. [Consultado en: julio de 2014].

Unesco (1997). ¿Qué universidad para el mañana? Hacia una evolución transdisciplinaria de la universidad. Declaración y recomendaciones del Congreso Internacional sobre Transdisciplinariedad, Locarno, Suiza.

Unesco (2005), Hacia las Sociedades del Conocimiento. [Online]. Disponible en: http://unesdoc.unesco.org/images/0014/001419/141908s.pdf. [Consultado: julio de 2011].

Schavino, Nancy y Villegas, Crisálida (2010). De la teoría a la praxis en el enfoque integrador transcomplejo. Congreso Iberoamericano de Educación Metas 2021, Buenos Aires, Argentina, 13-15 de septiembre.
Silva, Rosana (2014). La Transcomplejidad, un enfoque emergente para la producción de conocimiento complejo y transdisciplinario: La razón Tranversal. [Online]. Disponible en: http://es. calameo.com/read/0027843 7999ed5 f86529c. [Consultado: diciembre de 2014].

Soto, Mario (1999). Complejidad y sujeto humano. Tesis Doctoral, Universidad de Valladolid, Valladolid, España

Suñé, Albert (2004). El impacto de las barreras de aprendizaje en el rendimiento de las organizaciones. Tesis Doctoral No Publicada. Universitat Politècnica de Catalunya, Cataluña, España.

Velilla, Marco Antonio (2002). Manual de Iniciación Pedagógica al Pensamiento Complejo. Corporación para el Desarrollo Complexus/UNESCO, Editorial: Ediciones Jurídicas.

Vergara, Lisette (2010). Perspectiva de la Gerencia desde un Contexto Transcomplejo. [Online]. Disponible en: http:// epistemologiauba.blogspot.com/ 2010/11/perspectiva-de-la-gerenciadesde-un.html. [Consultado en julio de 2011].

Villegas, Crisálida (2006). La investigación: un enfoque Integrador transcomplejo. Maracay. Universidad Bicentenaria de Aragua, Vicerrectorado Académico. Decanato de Investigación, Extensión y Postgrado. 